\title{
The role of radiation therapy in the management of cutaneous melanoma
}

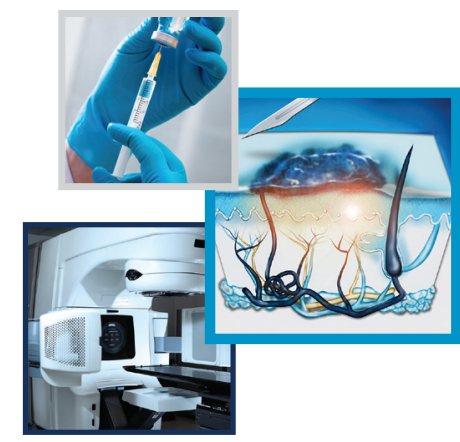

\author{
Charlene E Kan*,1 \& David B Mansur ${ }^{1}$
}

\section{Practice points}

- Surgical excision is the primary treatment for melanoma, and those that are $<1 \mathrm{~mm}$ thick are adequately treated with 1 $\mathrm{cm}$ margin. Melanomas $>1 \mathrm{~mm}$ thick are recommended to have surgical margins of $2 \mathrm{~cm}$, when anatomically possible.

- Risk factors for local recurrence include primary melanoma thickness (>4 $\mathrm{mm}$ ), the presence of ulceration and location at head and neck or extremity sites. Desmoplastic or desmoplastic neurotropic subtypes of melanoma may also be at higher risk of local recurrence.

- Adjuvant radiation to the primary site should be considered after wide local excision if surgical margins are inadequate and re-excision is not feasible. Patients with multiple high-risk features such as head and neck or distal extremity location, desmoplastic/desmoplastic neurotropic subtype, ulceration and tumors thicker than $4 \mathrm{~mm}$ should also be considered for radiation to the primary site.

- Patients with a melanoma of $\leq 1 \mathrm{~mm}$ thickness and clinically negative lymph nodes do not need any surgical workup for the lymph nodes given the very low risk of lymph node involvement. Patient with a melanoma $>1 \mathrm{~mm}$ should undergo a sentinel lymph node procedure.

- Patient with negative sentinel lymph nodes or submicrometastatic disease $(<0.1 \mathrm{~mm})$ can be observed. Patients with positive sentinel lymph nodes should undergo a completion lymph node dissection.

- Those who refuse initial sentinel lymph node procedure or completion lymph node dissection or are unable to undergo standard of care could consider radiation when the risk of positive lymph nodes is high based on the presence of positive sentinel lymph nodes or pathologic features of the primary melanoma.

- Patients with positive lymph nodes and high risk factors including extranodal extension, multiple involved nodes and large involved nodes $(>3 \mathrm{~cm})$ are at high risk of recurrence in the lymph node region and should consider adjuvant radiation to the nodal basin.

- There is no clear data indicating a benefit to hypofractionated regimens in treating melanoma. In the definitive treatment of patients, a total dose of 48-50 Gy in 2.4-2.5 Gy fractions for 20 fractions is recommended. In the setting of palliation, larger doses per fraction may be considered to shorten the length of treatment. Regimens such as $30 \mathrm{~Gy}$ in $3 \mathrm{~Gy}$ fractions for ten fractions or $20 \mathrm{~Gy}$ in $4 \mathrm{~Gy}$ fractions for five fractions would be recommended.

The local treatment of cutaneous melanoma with surgical excision is well-established; however, the role of adjuvant radiation is not well-defined. Characteristics of the primary melanoma can indicate a higher local recurrence risk and thus warrant consideration of adjuvant treatment to the primary site. Management of the lymph node regions has been studied in some recent clinical trials, especially with the advent of sentinel lymph node procedures. We will review the indications for radiation to the primary site as well as lymph node regions based on the available data.

First draft submitted: 20 August 2015; Accepted for publication: 6 November 2015; Published online: 24 February 2016

\footnotetext{
'Department of Radiation Oncology, Case Western Reserve University School of Medicine, University Hospitals, Seidman Cancer Center, 11100 Euclid Avenue, Lerner Tower B-141, Cleveland, OH 44106, USA

*Author for correspondence: Tel.: +1 216844 2517; Fax: +1 216844 4799; charlene.kan@uhhospitals.org
}

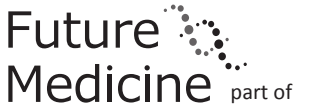




\section{KEYWORDS}

- melanoma - radiation therapy • sentinel lymph node
Cutaneous melanoma is a rare but deadly form of skin cancer. It is estimated that about 76,000 new cases of cutaneous melanoma will be diagnosed in 2014, accounting for only $4 \%$ of all skin cancers [1]. However, cutaneous melanoma accounts for about $75 \%$ of skin cancer deaths [2]. While early stage melanomas have an excellent prognosis, there is worsening survival as overall stage increases (Tables 1-3). According to Surveillance, Epidemiology, and End Results data, 5-year survival of those with localized melanoma was over $90 \%$ compared with $63 \%$ in those with regional lymph node (LN) disease and 16\% for those with distant metastatic disease [3]. Wide local excision (WLE) with or without sentinel lymph node (SLN) biopsy (SLNB) is the preferred initial treatment for cutaneous melanoma in medically operable patients. In general, radiation therapy (RT) has had a limited role in the treatment of melanoma, although it can be used in the adjuvant setting to treat the primary site and/ or LN regions and for palliation. Here, we will review the literature guiding recommendations for adjuvant external beam radiation treatment following initial surgical treatment of melanoma. We will first discuss the indications for postoperative radiation to the primary site and then address radiation to LN basins. We will consider the management of patients with negative LNs and those thought to have negative LNs but found to have positive LNs at the time of surgery. Then, we will discuss treatment recommendations for patients with clinically positive LNs. Recommended doses and fractionation will also be discussed. While radiation may be used in specific situations as primary treatment of melanoma or melanoma in situ, we will not discuss these particular situations, as surgery would be the standard treatment recommendation. In this review, we will not address the issues of treatment of in-transit disease, distant metastatic melanoma or stereotactic body radiation therapy.

Management of the primary site

- Indications for radiation to the primary site after wide local excision

Local control at the primary site is excellent with wide local excision $[4,5]$. Therefore, the role of RT in this setting has been limited. Nonetheless, adjuvant RT to the primary site has been advocated for those situations with a high risk of local recurrence [4-8].

Various randomized studies have examined the necessary surgical margins needed for melanomas based on depth of invasion. In general, melanomas $<1 \mathrm{~mm}$ thick can be adequately treated with $1 \mathrm{~cm}$ margins, while those that are $1.01-2 \mathrm{~mm}$ thick are treated with $1-2 \mathrm{~cm}$ margins, and those $>2 \mathrm{~cm}$ are recommended to have a margin of $2 \mathrm{~cm}$, if anatomically possible [5,9-13]. Using these surgical margins results in local recurrence rates of approximately $3 \%$. As the depth of invasion increases, the risk of local recurrence also increases. The Intergroup Melanoma Surgery Trial demonstrated that in melanomas $1-2 \mathrm{~mm}$ thick, the local recurrence rate was $2.3 \%$ compared with $11.7 \%$ in melanomas that were 3.01-4 mm thick [5]. However, additional studies on thicker melanomas demonstrate no additional benefit of larger margins. In a retrospective review, Heaton et al. demonstrated that for melanomas $>4 \mathrm{~mm}$ thickness, margins $>2 \mathrm{~cm}$ did not decrease the risk of local recurrence, which was approximately $12 \%$. In these patients, however, the risk of regional or distant recurrence was even greater at about $40 \%$ [14]. A large randomized UK study supports using margins $>1 \mathrm{~cm}$ for melanomas thicker than $2 \mathrm{~mm}$ [15]. Locoregional recurrences were statistically significantly higher in those undergoing surgery with a $1 \mathrm{~cm}$ margin compared with those with a $3 \mathrm{~cm}$ margin, hazard ratio (HR): 1.26, although overall survival (OS) was similar. The Swedish Melanoma Study Group demonstrated similar OS rates, whether $2 \mathrm{~cm}$ or $4 \mathrm{~cm}$ surgical margins were used in melanomas thicker than $2 \mathrm{~mm}$. They did find that local recurrences, although rare, occurred twice as often in the $2 \mathrm{~cm}$ surgical margin group, 4 versus $2 \%$, although this difference was not statistically significant $[16]$.

Ulceration is another factor that predicts for higher risk of local recurrence. In those with thick melanomas, the risk of local recurrence increased from about $10-16 \%$ if ulceration was present [14]. Even in melanomas $<4 \mathrm{~mm}$ in thickness, ulceration predicted for higher rates of local recurrence, $10.6 \%$ versus $1.5 \%$ with no ulceration [5]. Location of the primary site is also a factor that predicts for higher risk of local recurrence. Heaton et al. found that in thick melanomas local recurrence rates were highest for extremity lesions, $20 \%$, followed by head and neck lesions, $11 \%$, and then trunk lesions, 7\% [14]. Karakousis et al. found head and neck sites had the highest local recurrence rate at $9.4 \%$ followed by distal leg at $6.3 \%$, even after $2 \mathrm{~cm}$ surgical margins. All remaining sites: 
Table 1. TNM staging for cutaneous melanoma; American Joint Committee on Cancer 7th Edition.

\begin{tabular}{|ll|}
\hline Stage & Definition \\
\hline T stage: & Thickness $(\mathrm{mm}):$ \\
\hline- Tis & - None \\
- T1 & $-\leq 1.00$ \\
- T2 & $-1.01-2.00$ \\
- T3 & $-2.01-4.00$ \\
- T4 & $->4.00$ \\
\hline a: No ulceration; b: Ulceration present. & \\
\hline N stage: & Metastatic nodes (number): \\
\hline - N0 & -0 \\
\hline - N1 & -1 \\
\hline - N2 & $-2-3$ \\
\hline - N3 & $-4+$ or matted nodes or in-transit/satellites with \\
& metastatic nodes \\
\hline a: Micrometastases; b: Macrometastases. & \\
\hline M stage: & Site: \\
\hline- M0 & - None \\
\hline - M1a & - Distant skin, subcutaneous or nodal metastases \\
\hline - M1b & - Lung metastases \\
- M1c & - All other visceral metastases or elevated LDH \\
\hline LDH: Lactic acid dehydrogenase. & \\
Adapted with permission from [4]. & \\
\hline
\end{tabular}

trunk, distal arm, proximal arm and leg, had local recurrence rates of about $3 \%$ or less [5]. It appears that many of these high-risk features are additive. Patients with melanomas 3.1-4 mm thick on the trunk or proximal extremity had a recurrence rate of $4.1 \%$ compared with $23.1 \%$ to those on the head and neck or distal extremity [7].

Historic observation has noted that a particular subtype of melanoma seemed to have an increased propensity for local recurrence. The desmoplastic subtype was described as a bulky, subcutaneous tumor comprised of spindle cells and abundant collagen and could also have features of neurotropism, neural transformation or perineural or intraneural invasion $[17,18]$. Desmoplastic melanomas, including the neurotropic subgroup, tend to occur more in the head and neck region and in elderly males [19-22]. At diagnosis, they tend to have an increased depth of invasion compared with other melanomas, approximately 4-6 mm compared with $2 \mathrm{~mm}$; although this may be secondary to their often amelanotic appearance making it harder to diagnose $[20,23,24]$. Livestro et al. performed a case-matched review and found that stage III or IV disease was less common in desmoplastic/desmoplastic neurotropic melanomas compared with other melanomas and the risk of positive SLNs was also lower [25]. Thus, the desmoplastic subtype appears to be more locally aggressive and many institutional reports seemed to indicate a much higher rate of local recurrence compared with nondesmoplastic melanomas. Lens et al. performed a systematic review and reported that the local recurrence ranged from 6.7 to $56 \%$, while regional recurrence was $0-18 \%$ and systemic disease occurred $7.4-43.8 \%$ [21]. A small study of 59 patients revealed that for those patients with negative margins, the local recurrence rate was 24\% [24]. However, another group demonstrated low local recurrence rates with surgery alone. They noted that when $2 \mathrm{~cm}$ surgical margins were attempted for melanomas thicker than $2 \mathrm{~mm}$, and at least $1 \mathrm{~cm}$ margins for melanomas that were $<2 \mathrm{~mm}$, a local recurrence rate of $4 \%$ was observed [26]. Quinn et al. examined a group of desmoplastic/desmoplastic neurotropic melanoma patients and found that on logistic regression analysis, local recurrence rates were significantly higher in those that had $<1 \mathrm{~cm}$ excision margins compared with those with $>2 \mathrm{~cm}$ [22]. Thus, if adequate surgical margins can be obtained, desmoplastic and desmoplastic neurotropic melanomas may have similar local control rates after surgery. 


\begin{tabular}{|c|c|c|c|}
\hline Stages & T stage & N stage & M stage \\
\hline \multicolumn{4}{|c|}{ Clinical stage } \\
\hline 0 & Tis & No & MO \\
\hline IA & T1a & No & MO \\
\hline \multirow[t]{2}{*}{ IB } & $\mathrm{T} 1 \mathrm{~b}$ & No & Mo \\
\hline & $\mathrm{T} 2 \mathrm{a}$ & No & Mo \\
\hline \multirow[t]{2}{*}{$\| \mathrm{A}$} & $\mathrm{T} 2 \mathrm{~b}$ & No & Mo \\
\hline & T3a & No & Mo \\
\hline \multirow[t]{2}{*}{ IIB } & $\mathrm{T} 3 \mathrm{~b}$ & No & Mo \\
\hline & $\mathrm{T} 4 \mathrm{a}$ & No & Mo \\
\hline IIC & $\mathrm{T} 4 \mathrm{~b}$ & No & Mo \\
\hline III & Any $T$ & $\mathrm{~N}>\mathrm{NO}$ & MO \\
\hline IV & Any $T$ & Any N & M1 \\
\hline \multicolumn{4}{|c|}{ Pathologic stage } \\
\hline 0 & Tis & No & Mo \\
\hline IA & T1a & No & Mo \\
\hline \multirow[t]{2}{*}{ IB } & $\mathrm{T} 1 \mathrm{~b}$ & No & Mo \\
\hline & $\mathrm{T} 2 \mathrm{a}$ & No & Mo \\
\hline \multirow[t]{2}{*}{$\| \mathrm{A}$} & $\mathrm{T} 2 \mathrm{~b}$ & No & Mo \\
\hline & T3a & No & Mo \\
\hline \multirow[t]{2}{*}{ IIB } & $\mathrm{T} 3 \mathrm{~b}$ & No & Mo \\
\hline & $\mathrm{T} 4 \mathrm{a}$ & No & Mo \\
\hline IIC & $\mathrm{T} 4 \mathrm{~b}$ & NO & Mo \\
\hline \multirow[t]{2}{*}{ IIIA } & T1-4a & N1a & Mo \\
\hline & $\mathrm{T} 1-4 \mathrm{a}$ & $\mathrm{N} 2 \mathrm{a}$ & Mo \\
\hline \multirow[t]{9}{*}{ IIIB } & $\mathrm{T} 1-4 \mathrm{~b}$ & $\mathrm{~N} 1 \mathrm{a}$ & Mo \\
\hline & $\mathrm{T} 1-4 \mathrm{~b}$ & $\mathrm{~N} 2 \mathrm{a}$ & Mo \\
\hline & $\mathrm{T} 1-4 \mathrm{a}$ & $\mathrm{N} 1 \mathrm{~b}$ & Mo \\
\hline & $\mathrm{T} 1-4 \mathrm{a}$ & $\mathrm{N} 2 \mathrm{~b}$ & Mo \\
\hline & T1-4a & $\mathrm{N} 2 \mathrm{C}$ & Mo \\
\hline & $\mathrm{T} 1-4 \mathrm{~b}$ & N1b & Mo \\
\hline & $\mathrm{T} 1-4 \mathrm{~b}$ & $\mathrm{~N} 2 \mathrm{~b}$ & Mo \\
\hline & $\mathrm{T} 1-4 \mathrm{~b}$ & $\mathrm{~N} 2 \mathrm{C}$ & Mo \\
\hline & Any $T$ & N3 & Mo \\
\hline IIIC & Any $T$ & N2C & Mo \\
\hline IV & Any $T$ & N3 & M1 \\
\hline
\end{tabular}

However, as many of these occur in the head and neck region, it can be difficult to obtain adequate margins.

Due to the observation of increased local recurrences in desmoplastic and desmoplastic neurotropic melanomas, some institutions have used adjuvant RT to attempt to increase local control. A retrospective review of patients treated at University of California, Los Angeles (CA, USA) demonstrated a local recurrence rate of $48 \%$ after surgery with negative margins [27]. In the patients with recurrence, $66 \%$ received RT after surgical management and there were no recurrences subsequent to the RT. Recurrent patients who did not receive RT had a 57\% risk of another recurrence. In this review, a small number of patients received RT to gross disease as preoperative treatment, 45-50 Gy. In these patients, two had no viable disease on pathologic review and remained disease free at least 9 years after treatment. A third patient had positive margins at the time of surgery; however, despite no further treatment remained disease free 10 years later [27]. Chen et al. demonstrated similar local recurrence rates between their group of desmoplastic melanoma patients who received RT and those who did not, 7 versus $6 \%$, respectively [28]. The patients who received 
RT had significantly thicker tumors, deeper Clark level invasion, higher rates of ulceration and narrower margins, which may indicate that RT can increase local control when inadequate margins or other negative risk factors are present [28]. A retrospective review of 130 patients with nonmetastatic desmoplastic melanoma examined the local recurrence in patients that were observed after primary surgical resection compared with those who received adjuvant RT. In total, $93 \%$ of these patients had negative margins and $96 \%$ were node negative, but due to the desmoplastic pathology were thought to be at high risk for local recurrence. RT was most commonly delivered at 30 Gy in 6 Gy fractions for five fractions delivered twice a week. Local recurrence in the patients who did not receive RT was $24 \%$ compared with $7 \%$ in those who received RT [29]. A recent publication sought to determine which subgroup of patients with desmoplastic melanoma might have a significant benefit from radiation. They found that in their entire population, the local recurrence rate was $13 \%$; however, when comparing those who did or did not receive RT, the recurrence rate was 7 versus $17 \%$, respectively. Factors found to be statistically significant for local recurrence on multivariable analysis were male sex, Clark level $\mathrm{V}$ tumors, no adjuvant radiation and positive surgical resection margins [30]. Patients who had positive margins benefited the most from radiation, with a local recurrence rate of $54 \%$ if no RT was given compared with $14 \%$ if RT was received. Further analysis was done on the patients who had negative margins to determine if additional risk features might determine who may benefit from radiation. Using log-rank analysis, patients with a head and neck location, Breslow's depth $>4 \mathrm{~mm}$ or Clark level V tumor had significantly better local control with RT [30]. Table 4 provides a summary of the desmoplastic melanoma literature.

Long-term toxicity from radiation to the primary site is highly dependent on the locations being treated. Some of these side effects include wound healing issues, skin fibrosis, telangiectasias and skin pigment changes. Radiation to the head and neck region, an area often difficult to obtain adequate margins, can lead to additional long-term complications such as xerostomia, hypothyroidism and osteoradionecrosis. Based on the retrospective studies, risk of late complications from radiation can range up to $20 \%[29,30]$.
In summary, while some studies demonstrate an increased recurrence risk in those with desmoplastic/desmoplastic neurotropic melanomas others do not. These retrospective studies have mixed populations of patients and many variables that make it difficult to determine whether the observed increased recurrence rates in some studies are due to pathologic subtype or other factors. There is also no consistent benefit from RT in these retrospective studies; therefore, randomized trials are needed to determine whether or not desmoplastic histology is an independent risk factor for recurrence and whether or not RT will reduce this risk. There are currently two prospective randomized studies addressing adjuvant radiation for desmoplastic or desmoplastic neurotropic melanomas. The North Central Cancer Treatment Group Phase II study, N0275, randomizes patients with desmoplastic melanoma after excision with negative margins to observation or RT. This study was closed due to slow accrual and is currently in follow-up. Based on preliminary data describing perineural invasion, the neurotropic subgroup, as high risk for recurrence, the Australia and New Zealand Melanoma Trials Group and the Trans-Tasman Radiation Oncology Group (TROG) have initiated a Phase III trial, TROG 08.09, looking at adjuvant RT in patients with neurotropic melanoma of the head and neck [31]. Pending the results of the ongoing studies, it would be reasonable to offer radiation for patients with desmoplastic histology, particularly when recommended surgical margins are unable to be obtained [27-30].

In conclusion, first principles would support the use of adjuvant RT to the primary site after wide local excision if surgical margins are inadequate and re-excision is not feasible either for technical or medical reasons. While there is not yet any Phase III randomized evidence to guide management of the primary site after WLE of melanomas, it is reasonable to consider RT in those patients with high-risk features that may predispose to local recurrence. RT

\begin{tabular}{|c|c|}
\hline T stage & 10-year survival (\%) \\
\hline T1 & 92 \\
\hline T2 & 80 \\
\hline T3 & 63 \\
\hline $\mathrm{T} 4$ & 50 \\
\hline \multicolumn{2}{|c|}{ Data taken from [4]. } \\
\hline
\end{tabular}


Table 4. Literature summary of desmoplastic melanomas.

\begin{tabular}{|c|c|c|c|c|c|}
\hline Study (year) & Patients (n) & $\begin{array}{l}\text { Median } \\
\text { follow-up }\end{array}$ & Treatment & $\begin{array}{l}\text { Local } \\
\text { recurrence (\%) }\end{array}$ & Ref. \\
\hline Quinn et al. (1998) & 280 & - & Surgery ${ }^{\dagger}$ & 11 & {$[22]$} \\
\hline Jaroszewski et al. (2001) & 59 & - & Surgery $^{\dagger}$ & 39 & {$[24]$} \\
\hline Arora et al. (2005) & 49 & 3.7 years (mean) & Surgery & 4 & [26] \\
\hline \multirow[t]{2}{*}{ Vongtama et al. (2003) } & 44 & & Surgery & 48 & {$[27]$} \\
\hline & & & $\mathrm{RT}^{\ddagger}$ & 0 & \\
\hline \multirow[t]{2}{*}{ Chen et al. (2008) } & 101 & 3.4 years & Surgery & 6 & {$[28]$} \\
\hline & 27 & & Surgery $+\mathrm{RT}^{\S}$ & 7 & \\
\hline \multirow[t]{2}{*}{ Guadagnolo et al. (2014) } & 59 & 6.6 years & Surgery & 24 & [29] \\
\hline & 71 & & Surgery + RT & 7 & \\
\hline \multirow[t]{2}{*}{ Strom et al. (2014) } & 164 & 3.6 years & Surgery & 17 & {$[30]$} \\
\hline & 113 & & Surgery + $\mathrm{RT}^{\S}$ & 7 & \\
\hline
\end{tabular}

would therefore be recommended for patients with inadequate margins; $<1 \mathrm{~cm}$ for melanomas 1-2 mm thick and $<2 \mathrm{~cm}$ for melanomas $>2 \mathrm{~mm}$ in thickness. Patients with multiple high-risk features such as head and neck or distal extremity location, desmoplastic/desmoplastic neurotropic subtype, ulceration and tumors thicker than $4 \mathrm{~mm}$ should also be considered for radiation to the primary site.

Management of regional lymph nodes

- Patients with clinically negative lymph nodes

Patients with melanoma may present with no evidence of metastasis to $\mathrm{LN}$ regions based on clinical exam or imaging. However, with increasing thickness of the primary melanoma, the risk of LN positivity increases (Table 5). Management of clinically negative nodal regions continues to evolve. Historically, all patients with melanoma underwent complete lymph node dissection (LND); however, due to the significant toxicity associated with this operation, clinical trials sought to determine whether LND was indeed beneficial and also to determine whether only a subset of patients may benefit based on predictive factors. Possible approaches to patients with clinically negative LNs include LND versus SLNB versus RT versus observation.

The WHO Clinical Trial \# 14 sought to determine whether patients with clinically negative LNs would benefit from LND. They found that in patients with trunk melanomas of Breslow thickness $>1.5 \mathrm{~mm}$, there was a $22 \%$ rate of occult LN metastases in patients undergoing LND. In the group of patients that were randomized to the observation arm, 30\% developed regional nodal metastases as a first recurrence. OS at 5 years was $62 \%$ in patients who received immediate LND compared with $51 \%$ in patients who were initially observed, although this difference was not statistically significant. There was a significant difference in 5-year survival when comparing the group of patients who were found to be $\mathrm{LN}$ positive at the time of initial surgery with LND (50\%) versus those who had an LN recurrence and then subsequently underwent LND (20\%) [33]. Other groups have also reported that in the early stage melanoma population, where lymph nodes appear clinically negative, there does not appear to be a statistically significant difference in OS between immediate LND compared with delayed LND [34,35]. Cascinelli et al. demonstrated a survival difference between the immediate LND group of patients found to be LN positive compared with the observation group that eventually recurred in the regional LNs and underwent salvage surgery [33].

SLNB has become widely accepted as part of the workup for certain cancers and has been adopted in the management of cutaneous melanoma as a means of assessing lymph node regions with less morbidity than conventional LND. In early stage, clinically node negative, melanoma, about $20 \%$ of patients are found to have positive SLNs and of these patients only 20\% will be found to have additional positive LNs 
on completion LND $[33,36,37]$. Observation of nodal regions in high-risk cutaneous melanoma has not been widely adopted given the evidence for poor salvage after regional recurrence, and morbidity of regional recurrence, and the desire to identify LN positive patients for adjuvant systemic therapy options.

The MSLT-1 is a prospective clinical trial that randomized patients with intermediatethickness melanoma, $1.2-3.5 \mathrm{~mm}$, to wide local excision plus observation versus wide local excision and SLNB. Patients found to have a positive SLN went on to completion LND. Patients with intermediate thickness melanomas in the observation arm had $15 \%$ nodal recurrence as first site of recurrence compared with $5 \%$ in those who underwent SLNB. Those with thick melanomas, $>3.5 \mathrm{~mm}$, had a $9 \%$ first recurrence in nodal region if they had undergone SLNB compared with $34 \%$ in the observation arm. The 10 -year disease-free survival was significantly higher in the SLNB group (71\%) compared with the observation group $(64 \% ; \mathrm{p}=0.01)$. However, melanoma-specific survival at 5 and 10 years was similar. When comparing those found to have a positive SLN to those in the observation group that subsequently developed nodal recurrence, the 5 -year overall survival was $72 \%$ compared with $52 \%$, respectively $(p=0.004)$. In intermediate-thickness melanomas, the 10-year melanoma-specific survival of those with lymph node metastases was $62 \%$ in the SLNB arm compared with $42 \%$ in the observation arm ( $\mathrm{p}=0.006)$, but no significant difference was noted in nodepositive patients with thick melanomas $[38,39]$. MSLT-1 demonstrates that the use of SLNB to determine which patients to take for completion lymphadenectomy may have some disease-specific survival advantage compared with delayed lymphadenectomy and may spare some patients the morbidity associated with LND. Table 6 summarizes the literature examining observation versus immediate surgical management of the $\mathrm{LN}$ regions.

Lee et al. published a retrospective study examining factors associated with additional positive LNs after positive SLN in melanoma patients with clinical stage I-II tumors. In their overall population, they noted approximately $20 \%$ of patients had positive SLNs. They identified a population that had clinicopathologic information available for analysis after completion LND. About $24 \%$ of patients were found to have additional positive LNs at the time of completion LND and $2 \%$ had positive nonsentinel LNs resected at the time of lymphatic mapping/sentinel lymphadenectomy [40]. In multivariate analysis, Breslow thickness $(>3 \mathrm{~mm})$ and SLN metastasis size $(>2 \mathrm{~mm}$ ) were significantly associated with additional nonsentinel $L N$ positivity. Based on their cohort, having neither of the risk factors led to a $12.3 \%$ chance of having additional positive LNs compared with $40 \%$ if both risk factors were present. A study from The Netherlands examined different pathologic characteristics of the positive SLN to determine whether a subpopulation of patients could avoid completion LN dissection. They concluded that if the metastasis was $<0.1 \mathrm{~mm}$, the risk of additional positive LNs was extremely rare and 5-year overall survival was $100 \%$; therefore, these patients could be treated as LN negative and did not require completion lymphadenectomy. On multivariate analysis, larger SLN tumor size and ulceration at the primary site were significant independent prognostic factors for worse disease-free survival [36]. These studies suggest that some patients with positive SLNs may not need to undergo further therapy.

Scoggins et al. reported that in their analysis of melanoma patients with primary tumors thicker than $4 \mathrm{~mm}$, patients with positive SLNs who underwent completion LND had $0 \%$ recurrence in the nodal basin compared with $13 \%$ in those who had negative SLNs, and thus no additional LN dissection $(\mathrm{p}=0.01)$ [41]. Based on data from MSLT-1 showing $88 \%$ of patients with only one positive SLN have no further LNs involved on completion LND, the MSLT2 trial is seeking to determine in patients with positive SLNs, what subgroup would benefit from immediate completion LND versus close surveillance. Patients not undergoing immediate LND will be closely monitored with high-definition ultrasonography in order to detect any early LN recurrences (Figure 1) [42]. This trial has completed accrual and is awaiting maturation of the data.

\begin{tabular}{|c|c|}
\hline $\begin{array}{l}\text { Tumor thickness } \\
\text { (mm) }\end{array}$ & $\begin{array}{l}\text { Lymph node positive } \\
\text { (\%) }\end{array}$ \\
\hline$\leq 1.00$ & 4 \\
\hline $1.01-2.00$ & 14 \\
\hline $2.01-4.00$ & 29 \\
\hline$>4.00$ & 45 \\
\hline \multicolumn{2}{|c|}{ Adapted with permission from [32]. } \\
\hline
\end{tabular}


Table 6. Literature summary of surgical management of clinically negative lymph nodes in stage I-II melanoma.

\begin{tabular}{|c|c|c|c|c|c|c|}
\hline Study & Treatment & $\begin{array}{l}\text { Mean follow-up } \\
\text { (years) }\end{array}$ & Patients (n) & 5-year OS & $\mathrm{LN}+^{+}$ & Ref. \\
\hline \multirow[t]{2}{*}{ Cascinelli et al. (1998) } & Delayed nodal dissection & 11 & 118 & $51 \%^{\ddagger}$ & $30 \%$ & [33] \\
\hline & Elective node dissection & & 122 & $61 \%$ & $22 \%$ & \\
\hline \multirow[t]{2}{*}{ Balch et al. (1996) } & Observation & 7.4 & 361 & $82 \%^{\ddagger}$ & & [34] \\
\hline & Elective node dissection & & 379 & $86 \%^{\S}$ & & \\
\hline \multicolumn{6}{|l|}{ Sim et al. (1986) } & [35] \\
\hline \multicolumn{7}{|c|}{ 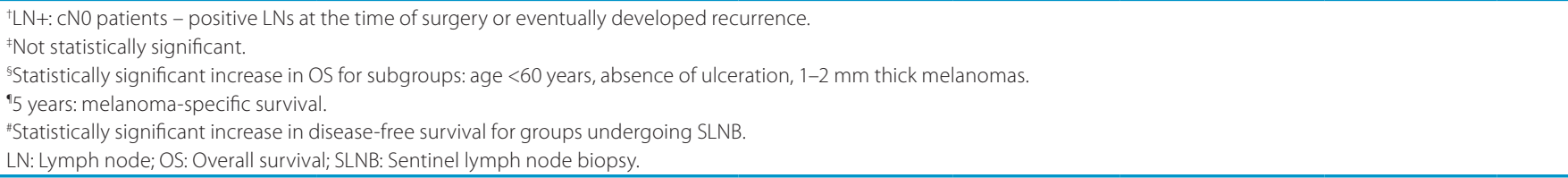 } \\
\hline
\end{tabular}

An area of further research would include determining whether RT could substitute for LND in patients with a small number of positive SLNs. In the situation where a patient refuses an SLNB but has a primary melanoma $>1 \mathrm{~mm}$, with a $>15 \%$ chance of positive LNs, RT might be considered to increase regional control. In patients with a known positive SLN but have a contraindication to completion LND or who refuses $\mathrm{LND}$, a discussion regarding the possible benefit of RT may be warranted.

Use of RT as definitive treatment of the regional nodes could be considered in specific clinical scenarios. Patients with high risk of nodal involvement should be considered for RT if surgical lymph node procedures are refused, or if the patient is considered medically inoperable.

For those patients with a positive SLN metastasis $<0.1 \mathrm{~mm}$ (submicrometastases), the risk of additional positive lymph nodes was $0 \%$ in the study by van Akkooi and thus these patients may not need any further treatment after WLE and SLND [36].

In summary, managing melanoma patients with clinically negative LNs depends on the thickness of the melanoma. Those with a melanoma of less than or equal to $1 \mathrm{~mm}$ thickness have a very low risk of LN involvement, less than 5\%, and can forego any further surgical procedures for LN workup. Given the morbidity associated with an LND, the recommended procedure for those with a melanoma $>1 \mathrm{~mm}$ would be an SLNB. Those with negative SLNs have a low risk of nodal recurrence and thus can be observed. In those patients who are found to have pathologically positive SLNs, the standard treatment is completion LND. The use of RT in patients who do not undergo an LND has not been studied but could be considered in certain circumstances when the risk of positive LNs is high based on the presence of positive SLNs or pathologic features of the primary melanoma. Further management of patients with positive LNs will be discussed in the next section.

\section{- Management of patients with clinically positive lymph nodes}

Unfortunately, some patients with newly diagnosed melanoma will present with enlarged LNs while other patients will have recurrence only in the adjacent lymph node region, with no signs of distant metastatic disease. In patients with known LN metastasis, whether by clinical exam or imaging, many clinical trials have examined whether postoperative RT can decrease localregional recurrences. Multiple studies have also sought to determine risk factors predicting recurrence in the nodal basin. After lymphadenectomy alone, regional relapse rates occur in up to $50 \%$ of patients [43], therefore adjuvant radiation has been explored as a way to decrease the risk of recurrence.

Multiple retrospective studies have been reported comparing the effectiveness of adjuvant RT after LND in patients with positive LNs. For example, Ballo et al. reported on the MD Anderson Cancer Center (MDACC) experience where melanoma patients with involved $\mathrm{LN}$ and risk factors for recurrence were treated with adjuvant radiation after lymphadenectomy. Most of the patients had risk factors such as extracapsular extension, $\mathrm{LN}$ size $>3 \mathrm{~cm}$, four or more involved 
LNs, or recurrent disease after previous lymphadenectomy. At 5 years, the regional, in basin, control rate was excellent at $89 \%$ [44]. The first randomized prospective trial to examine the issue of postoperative RT to nodal regions was published by Creagan in 1978. In this small study of 56 patients, those with truncal, extremity or unknown primary melanoma were randomized to observation versus radiation after positive nodes were found on lymphadenectomy. Radiation was delivered for a total of $5000 \mathrm{rad}$ in 28 fractions of $178 \mathrm{rad}$ to the tumor midplane, in a split course manner with a 3-4-week break after 14 fractions. The median time to recurrence following RT was 20 months compared with 9 months following surgery alone, although this was not statistically significant. After RT, median survival was 33 versus 22 months for surgery alone, but again this was not statistically significant. It was noted that the two groups, although randomized, were not balanced for age and nodal distributions and thus covariate analysis was performed. The study concluded that treatment did not have a significant effect upon survival or disease-free interval and that factors such as age and sex were significant in predicting for survival while the number of positive nodes was associated with disease-free interval [45]. However, given the very small number of patients these trends are intriguing.

More recently, prospective studies have been published examining the benefit of adjuvant radiation in the setting of positive LNs. TROG 96.06, a Phase II study, treated patients with pathologically proven melanoma in the lymph nodes or extranodal soft tissues in the lymph node basin with risk factors for recurrence based on greater than one LN involved, histological evidence of extranodal spread, tumor spill or resection of a recurrence after previous surgery. After macroscopic resection, patients were treated with $48 \mathrm{~Gy}$ in $2.4 \mathrm{~Gy}$ fractions for 20 fractions. If the patient was thought to have positive margins, total dose could be increased to $50 \mathrm{~Gy}$. Regional in-field relapse rate was $6.8 \%$ [46] compared with previous rates of local-regional control after surgery alone of about $30-40 \%$ leading to the conclusion that postoperative RT may decrease locoregional

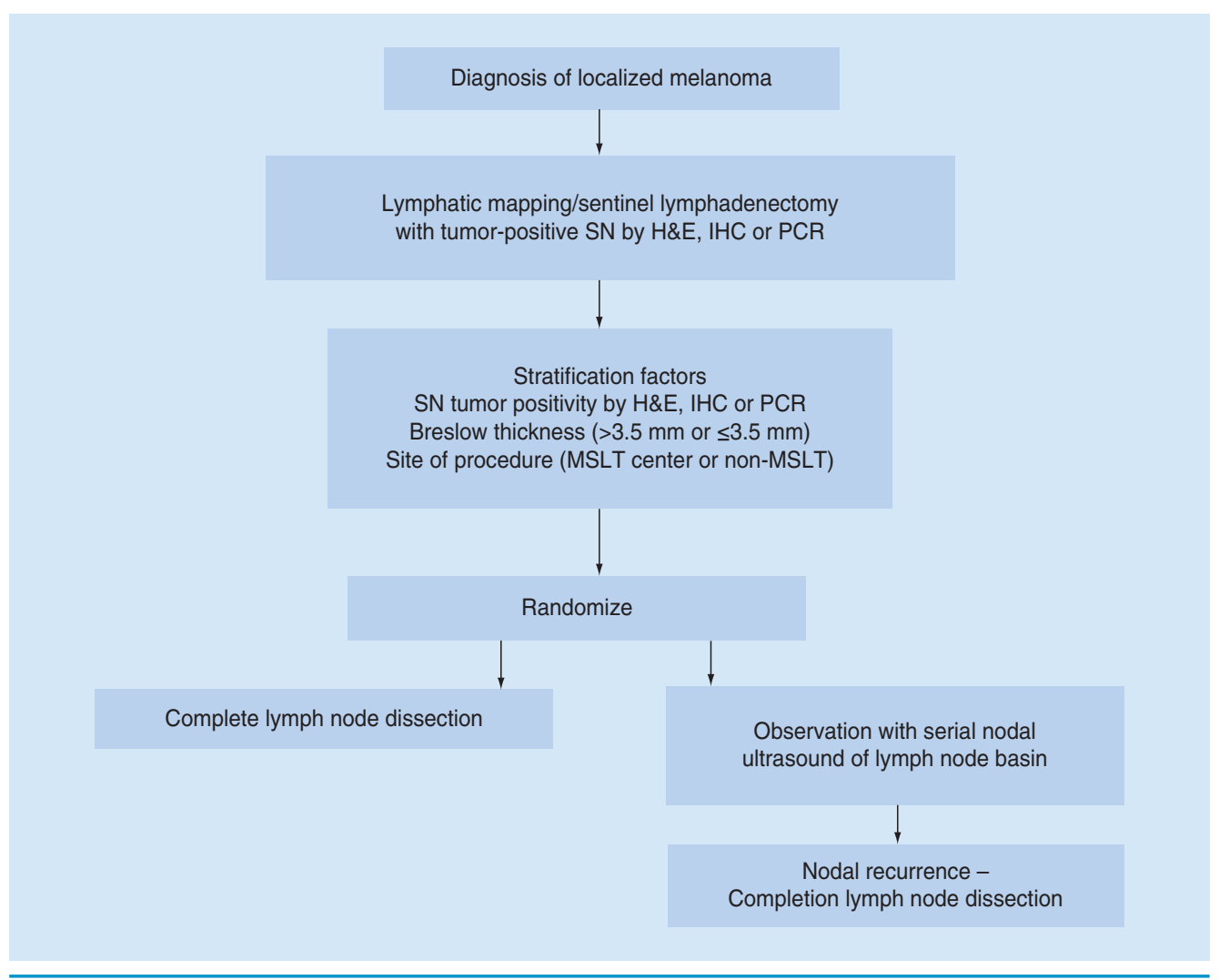

Figure 1. Treatment schema for MSLT-2.

H\&E: Hematoxylin and eosin stain; IHC: Immunohistochemistry; SN: Sentinel node.

Adapted with permission from [39]. 
recurrence. Additionally, late toxicity was limited to $10-20 \%$ grade 3 toxicity depending on the lymph node region treated. These data resulted in TROG 02.01, a Phase III study, in patients at high risk of $\mathrm{LN}$ relapse after therapeutic LAD.

TROG 02.01, is a prospective, randomized, trial in which patients with clinically evident, palpable, LN involvement, confirmed pathologically at the time of surgery, were randomized to observation or treated with RT, 48 Gy in 2.4 Gy fractions for 20 fractions. Inclusion criteria were presence of extranodal tumor spread, nodal size greater than $3 \mathrm{~cm}$ (cervical nodes) or $4 \mathrm{~cm}$ (axillary and inguinal nodes), one or more involved parotid nodes, two or more involved cervical or axillary nodes or three or more involved inguinal nodes. The cumulative incidence of LN relapse at 3 years was $31 \%$ in the observation group compared with $19 \%$ in the RT group with a HR of 0.56 , a statistically significant difference. There was not a significant difference in recurrence-free survival, OS or rate of distant recurrence, although, this study had a number of ineligible patients and was not powered to detect an OS benefit. On multivariable analysis, extranodal spread was the only independent risk factor for predicting LN relapse [47]. The most common early grade $3-4$ toxicities related to radiation treatment were dermatitis and pain in the area of treatment. Long-term toxicity and quality of life outcomes are still to be reported.

Radiation to the nodal basins is not without side effects. The location receiving radiation dictates the possible toxicities. Common late toxicities include skin and subcutaneous changes, bone and joint changes, nerve damage and lymphedema. The rates of skin/subcutaneous changes, bone/joint changes and nerve damage are very low, $<5 \%$ [46]. Lymphedema rates vary from about 10 to $30 \%$ depending on the location being radiated [44-46], although the rate of lymphedema with surgery alone can be as high as $20 \%[45]$.

In summary, based on retrospective studies as well as this prospective randomized study, adjuvant RT in melanoma patients with positive LNs with high-risk features can significantly decrease the risk of local-regional recurrence; however, OS is not affected. Extranodal extension, multiple involved nodes and large involved nodes $(>3 \mathrm{~cm})$ appear to be the strongest factors associated with recurrence in the nodal basin [48] and thus warrant consideration of adjuvant RT. These same criteria may be applied to patients who were initially thought to be LN negative but are found to have positive lymph node after SLNB and LND.

\section{Dose \& fractionation}

Many lines of evidence indicate melanoma is a relatively radioresistant malignancy. This led to the belief that larger fraction sizes are required when treating with RT. There is some evidence that higher dose per fraction may be more effective, as reported in an analysis by MDACC. In a retrospective study, they found that doses larger than 4-5 Gy per fraction yielded better local control than doses less than 4-5 Gy [49,50]. Two hypofractionated regimens were examined in a randomized study treating metastatic or inoperable recurrent melanoma patients. Both regimens were delivered twice a week: $27 \mathrm{~Gy}$ was delivered in three fractions of 9 Gy compared with $40 \mathrm{~Gy}$ in eight fractions of $5 \mathrm{~Gy}$. Tumor response was similar with about $70 \%$ of tumors having a complete response and $97 \%$ having some overall response to treatment. The toxicity was also similar in both regimens [50]. The RTOG conducted a trial to evaluate different fractionation regimens. They treated patients with measurable lesions for a total dose of $32 \mathrm{~Gy}$ in four fractions of $8 \mathrm{~Gy}$, once a week, or $20 \mathrm{~Gy}$ in eight fractions of $2.5 \mathrm{~Gy}$. They noted no significant difference in response rates with a complete remission rate of about $24 \%$ and a partial response of approximately $35 \%$ [51]. The early toxicity was slightly greater, but not significantly, in the hypofractionated arm. Regardless of the specific fractionation, there are significant response rates in these analyses indicating the clinical usefulness of RT in treating this disease in both the curative or palliative setting. This question of relative radiation resistance of melanoma has been reviewed in detail [52].

The limited existing data indicates no clear benefit to the hypofractionated regimens. Given the potential increased risk of toxicities with larger fraction size, in definitive treatment or in patients who may have extended survival a total dose of 48-50 Gy in 2.4-2.5 Gy fractions seems appropriate. The above studies also indicate that radiation alone can be effective in the setting of gross disease requiring palliation. In the setting of palliation using larger doses per fraction may be beneficial for the patient in terms of shortening length of treatment. Regimens such as $30 \mathrm{~Gy}$ in $3 \mathrm{~Gy}$ fractions for ten fractions or 20 Gy in 4 Gy fractions for five fractions would be recommended. 


\section{Conclusion \& future perspective}

Melanoma, when diagnosed and treated early can have very favorable outcomes. However, patients with more advanced primary tumors or involvement of the lymph nodes with high-risk factors have decreased survival and many may benefit from adjuvant treatment after surgery. We have reviewed the current data that guides decisions to use radiation as adjuvant treatment. We await the results of the ongoing trials such as N0275, TROG 08.09 and MSLT2 for further guidance on how to manage the primary site and nodal regions. Treatment of melanoma has evolved quickly over the last few years. The advent of effective immunotherapies and other targeted therapies has already significantly improved outcomes. It will be of interest to investigate the combination of these therapies with radiation. While caution will need to be taken to avoid excess toxicities, there is a great potential for combining these different modalities of treatment to improve patient survival. Additionally, the area of tumor genomics is an area of very active research. Improvement in technology can now allow a patient's tumor to be sequenced and may allow for individualized treatment based on the mutations and aberrations that are present.

Financial \& competing interests disclosure The authors have no relevant affiliations or financial involvement with any organization or entity with a financial interest in or financial conflict with the subject matter or materials discussed in the manuscript. This includes employment, consultancies, honoraria, stock ownership or options, expert testimony, grants or patents received or pending, or royalties.

No writing assistance was utilized in the production of this manuscript.

\section{References}

Papers of special note have been highlighted as:

-• of considerable interest

1 Siegel R, Ma J, Zou Z, Jemal A. Cancer statistics, 2014. CA Cancer J. Clin. 64(1), 9-29 (2014).

2 Jemal A, Siegel R, Ward E, Hao Y, Xu J, Thun MJ. Cancer statistics, 2009. CA Cancer J. Clin. 59(4), 225-249 (2009).

3 American Cancer Society. Cancer facts \& figures, 2009. Atlanta: American Cancer Society (2009).

4 Balch CM, Gershenwald JE, Soong SJ et al. Final version of 2009 AJCC melanoma staging and classification. J. Clin. Oncol. 27(36), 6199-6206 (2009).

5 Karakousis CP, Balch CM, Urist MM, Ross MM, Smith TJ, Bartolucci AA. Local recurrence in malignant melanoma: long-term results of the multi-institutional randomized surgical trial. Ann. Surg. Oncol. 3(5), 446-452 (1996).

-• Reports on the efficacy of $2 \mathrm{~cm}$ resection margins for melanomas 1-4 mm thick.

6 Mansson-Brahme E, Carstensen J, Erhardt K, Lagerlof B, Ringborg U, Rutqvist LE. Prognostic factors in thin cutaneous malignant melanoma. Cancer 73(9), 2324-2332 (1994).

7 Balch CM, Soong SJ, Smith T et al. Long-term results of a prospective surgical trial comparing $2 \mathrm{~cm}$ vs. $4 \mathrm{~cm}$ excision margins for 740 patients with 1-4 mm melanomas. Ann. Surg. Oncol. 8(2), 101-108 (2001).
8 Urist MM, Balch CM, Soong S, Shaw HM, Milton GW, Maddox WA. The influence of surgical margins and prognostic factors predicting the risk of local recurrence in 3445 patients with primary cutaneous melanoma. Cancer 55(6), 1398-1402 (1985).

9 Balch CM, Urist MM, Karakousis CP et al. Efficacy of $2 \mathrm{~cm}$ surgical margins for intermediate-thickness melanomas (1 to $4 \mathrm{~mm}$ ). Results of a multi-institutional randomized surgical trial. Ann. Surg. 218(3), 262-267 (1993).

10 Cohn-Cedermark G, Rutqvist LE, Andersson $\mathrm{R}$ et al. Long term results of a randomized study by the Swedish Melanoma Study Group on 2-cm versus 5-cm resection margins for patients with cutaneous melanoma with a tumor thickness of 0.8-2.0 mm. Cancer 89(7), 1495-1501 (2000).

- The multicenter trial demonstrates the adequacy of $2 \mathrm{~cm}$ resection margins for melanomas $<2 \mathrm{~mm}$ in thickness.

11 Khayat D, Rixe O, Martin G et al. Surgical margins in cutaneous melanoma $(2 \mathrm{~cm}$ versus $5 \mathrm{~cm}$ for lesions measuring less than $2.1 \mathrm{~mm}$ thick). Cancer 97(8), 1941-1946 (2003).

12 Veronesi U, Cascinelli N. Narrow excision (1 cm margin). A safe procedure for thin cutaneous melanoma. Arch. Surg. 126(4), 438-441 (1991).

13 Veronesi U, Cascinelli N, Adamus J et al. Thin stage I primary cutaneous malignant melanoma. Comparison of excision with margins of 1 or $3 \mathrm{~cm}$. N. Engl. J. Med. 318(18), 1159-1162 (1988).

14 Heaton KM, Sussman JJ, Gershenwald JE et al. Surgical margins and prognostic factors in patients with thick $(>4 \mathrm{~mm}$ ) primary melanoma. Ann. Surg. Oncol. 5(4), 322-328 (1998).

15 Thomas JM, Newton-Bishop J, A'hern R et al. Excision margins in high-risk malignant melanoma. N. Engl. J. Med. 350(8), 757-766 (2004).

16 Gillgren P, Drzewiecki KT, Niin M et al. 2 -cm versus $4-\mathrm{cm}$ surgical excision margins for primary cutaneous melanoma thicker than $2 \mathrm{~mm}$ : a randomised, multicentre trial. Lancet 378(9803), 1635-1642 (2011).

-. The multicenter trial demonstrates the adequacy of $2 \mathrm{~cm}$ resection margins for melanomas $>2 \mathrm{~mm}$ in thickness.

17 Conley J, Lattes R, Orr W. Desmoplastic malignant melanoma (a rare variant of spindle cell melanoma). Cancer 28(4), 914-936 (1971).

18 Reed RJ, Leonard DD. Neurotropic melanoma. A variant of desmoplastic melanoma. Am. J. Surg. Pathol. 3(4), 301-311 (1979).

19 Carlson JA, Dickersin GR, Sober AJ, Barnhill RL. Desmoplastic neurotropic melanoma. A clinicopathologic analysis of 28 cases. Cancer 75(2), 478-494 (1995).

20 Feng Z, Wu X, Chen V, Velie E, Zhang Z. Incidence and survival of desmoplastic melanoma in the United States, 1992-2007. J. Cutan. Pathol. 38(8), 616-624 (2011). 
21 Lens MB, Newton-Bishop JA, Boon AP. Desmoplastic malignant melanoma: a systematic review. Br. J. Dermatol. 152(4), 673-678 (2005).

22 Quinn MJ, Crotty KA, Thompson JF, Coates AS, O’Brien CJ, McCarthy WH. Desmoplastic and desmoplastic neurotropic melanoma: experience with 280 patients. Cancer 83(6), 1128-1135 (1998).

23 Hawkins WG, Busam KJ, Ben-Porat L et al. Desmoplastic melanoma: a pathologically and clinically distinct form of cutaneous melanoma. Ann. Surg. Oncol. 12(3), 207-213 (2005).

24 Jaroszewski DE, Pockaj BA, Dicaudo DJ, Bite $\mathrm{U}$. The clinical behavior of desmoplastic melanoma. Am. J. Surg. 182(6), 590-595 (2001).

25 Livestro DP, Muzikansky A, Kaine EM et al. Biology of desmoplastic melanoma: a case-control comparison with other melanomas. J. Clin. Oncol. 23(27), 6739-6746 (2005).

26 Arora A, Lowe L, Su L et al. Wide excision without radiation for desmoplastic melanoma. Cancer 104(7), 1462-1467 (2005).

27 Vongtama R, Safa A, Gallardo D, Calcaterra T, Juillard G. Efficacy of radiation therapy in the local control of desmoplastic malignant melanoma. Head Neck 25(6), 423-428 (2003).

28 Chen JY, Hruby G, Scolyer RA et al. Desmoplastic neurotropic melanoma: a clinicopathologic analysis of 128 cases. Cancer 113(10), 2770-2778 (2008).

29 Guadagnolo BA, Prieto V, Weber R, Ross MI, Zagars GK. The role of adjuvant radiotherapy in the local management of desmoplastic melanoma. Cancer 120(9), 1361-1368 (2014).

30 Strom T, Caudell JJ, Han D et al. Radiotherapy influences local control in patients with desmoplastic melanoma. Cancer 120(9), 1369-1378 (2014).

31 Foote MC, Burmeister B, Burmeister E, Bayley G, Smithers BM. Desmoplastic melanoma: the role of radiotherapy in improving local control. ANZ J. Surg. 78(4), 273-276 (2008).

32 Bonnen MD, Ballo MT, Myers JN et al. Elective radiotherapy provides regional control for patients with cutaneous melanoma of the head and neck. Cancer 100(2), 383-389 (2004).

33 Cascinelli N, Morabito A, Santinami M, Mackie RM, Belli F. Immediate or delayed dissection of regional nodes in patients with melanoma of the trunk: a randomised trial. WHO Melanoma Programme. Lancet 351(9105), 793-796 (1998).

- Reports an improved survival for immediate lymph node (LN) dissection in those with positive LNs compared with delayed LN dissection.

34 Balch CM, Soong SJ, Bartolucci AA et al. Efficacy of an elective regional lymph node dissection of 1 to $4 \mathrm{~mm}$ thick melanomas for patients 60 years of age and younger. Ann. Surg. 224(3), 255-263 (1996).

-• Reported long-term results demonstrating improved survival for patients with nonulcerated melanomas undergoing elective LN dissection compared with observation.

35 Sim FH, Taylor WF, Pritchard DJ, Soule EH. Lymphadenectomy in the management of stage I malignant melanoma: a prospective randomized study. Mayo Clin. Proc. 61(9), 697-705 (1986).

36 Van Akkooi AC, De Wilt JH, Verhoef C et al. Clinical relevance of melanoma micrometastases $(<0.1 \mathrm{~mm})$ in sentinel nodes: are these nodes to be considered negative? Ann. Oncol. 17(10), 1578-1585 (2006).

37 Mcmasters KM, Wong SL, Edwards MJ et al. Frequency of nonsentinel lymph node metastasis in melanoma. Ann. Surg. Oncol. 9(2), 137-141 (2002).

38 Morton DL, Thompson JF, Cochran AJ et al. Sentinel-node biopsy or nodal observation in melanoma. N. Engl. J. Med. 355(13), 1307-1317 (2006).

39 Morton DL, Thompson JF, Cochran AJ et al. Final trial report of sentinel-node biopsy versus nodal observation in melanoma. N. Engl. J. Med. 370 (7), 599-609 (2014).

-• Reported 10-year data on a Phase III demonstrating the effectiveness of doing sentinel LN biopsy and completion lymphadenectomy in those with positive sentinel LNs.

40 Lee JH, Essner R, Torisu-Itakura H, Wanek L, Wang H, Morton DL. Factors predictive of tumor-positive nonsentinel lymph nodes after tumor-positive sentinel lymph node dissection for melanoma. J. Clin. Oncol. 22(18), 3677-3684 (2004).

41 Scoggins CR, Bowen AL, Martin RC 2nd et al. Prognostic information from sentinel lymph node biopsy in patients with thick melanoma. Arch. Surg. 145(7), 622-627 (2010).

42 Morton DL. Overview and update of the Phase III Multicenter Selective Lymphadenectomy Trials (MSLT-I and MSLT-II) in melanoma. Clin. Exp. Metastasis 29(7), 699-706 (2012).

43 Lee RJ, Gibbs JF, Proulx GM, Kollmorgen DR, Jia C, Kraybill WG. Nodal basin recurrence following lymph node dissection for melanoma: implications for adjuvant radiotherapy. Int. J. Radiat. Oncol. Biol. Phys. 46(2), 467-474 (2000).

44 Ballo MT, Ross MI, Cormier JN et al. Combined-modality therapy for patients with regional nodal metastases from melanoma. Int. J. Radiat. Oncol. Biol. Phys. 64(1), 106-113 (2006).

45 Creagan ET, Cupps RE, Ivins JC et al. Adjuvant radiation therapy for regional nodal metastases from malignant melanoma: a randomized, prospective study. Cancer 42(5), 2206-2210 (1978).

46 Burmeister BH, Mark Smithers B, Burmeister E et al. A prospective Phase II study of adjuvant postoperative radiation therapy following nodal surgery in malignant melanoma-Trans Tasman Radiation Oncology Group (TROG) Study 96.06. Radiother. Oncol. 81(2), 136-142 (2006).

47 Burmeister BH, Henderson MA, Ainslie J et al. Adjuvant radiotherapy versus observation alone for patients at risk of lymph-node field relapse after therapeutic lymphadenectomy for melanoma: a randomised trial. Lancet Oncol. 13(6), 589-597 (2012).

- Reported on a Phase III trial of observation compared with adjuvant radiation after therapeutic lymphadenectomy demonstrating a significant decrease in $\mathrm{LN}$ field relapses.

48 Bastiaannet E, Beukema JC, Hoekstra HJ. Radiation therapy following lymph node dissection in melanoma patients: treatment, outcome and complications. Cancer Treat. Rev. 31(1), 18-26 (2005).

49 Bentzen SM, Overgaard J, Thames HD et al. Clinical radiobiology of malignant melanoma. Radiother. Oncol. 16(3), 169-182 (1989).

50 Overgaard J, Overgaard M, Hansen PV, von der Maase $\mathrm{H}$. Some factors of importance in the radiation treatment of malignant melanoma. Radiother. Oncol. 5(3), 183-192 (1986).

51 Sause WT, Cooper JS, Rush S et al. Fraction size in external beam radiation therapy in the treatment of melanoma. Int. J. Radiat. Oncol. Biol. Phys. 20(3), 429-432 (1991).

- Reported on RTOG 83-05 demonstrating no difference between hypofractionated compared with a more conventional fractionation schedule with regard to complete and partial remissions.

52 Stevens G, Thompson JF, Firth I, O'Brien CJ, McCarthy WH, Quinn MJ. Locally advanced melanoma: results of postoperative hypofractionated radiation therapy. Cancer 88(1), 88-94 (2000). 